\title{
Biomarker Testing Rates in Patients with Advanced Non-Small Cell Lung Cancer Treated in the Community
}

\author{
Eric Nadler ${ }^{1 *}$, Melissa Pavilack ${ }^{2}$, Jamyia Clark ${ }^{3}$, Janet Espirito ${ }^{3}$, Ancilla Fernandes ${ }^{2}$ \\ ${ }^{1}$ US Oncology Health Informatics and Internet Technology, Texas Oncology-Baylor Charles A. Sammons Cancer Center, Dallas, \\ TX, US \\ ${ }^{2}$ Health Economics and Outcomes Research, AstraZeneca US, Gaithersburg, MD, US \\ ${ }^{3}$ Real World Evidence, McKesson Life Sciences, The Woodlands, TX, US \\ Email: *eric.nadler@usoncology.com
}

How to cite this paper: Nadler, E., Pavilack, M., Clark, J., Espirito, J. and Fernandes, A. (2019) Biomarker Testing Rates in Patients with Advanced Non-Small Cell Lung Cancer Treated in the Community. Journal of Cancer Therapy, 10, 971-984. https://doi.org/10.4236/jct.2019.1012083

Received: November 15, 2019

Accepted: December 20, 2019

Published: December 23, 2019

Copyright $\odot 2019$ by author(s) and Scientific Research Publishing Inc. This work is licensed under the Creative Commons Attribution International License (CC BY 4.0).

http://creativecommons.org/licenses/by/4.0/

\begin{abstract}
Introduction: Over the past few years, molecular targeted therapies have been emerging for the treatment of metastatic non-small cell lung cancer (NSCLC). Targeted therapy is associated with improved outcomes in patients with identified gene alterations, and national guidelines recommend routine biomarker testing. This study evaluated real-world rates of documented epidermal growth factor receptor (EGFR) mutation and other biomarker testing in patients with advanced NSCLC over time. Methods: Adult patients with Stage IV NSCLC were identified between January 1, 2012 and May 31, 2017 from the US Oncology Network iKnowMed ${ }^{\mathrm{TM}}$ electronic health records. Patients were examined overall and by histology. Rates of documented EGFR mutation and other biomarker testing were calculated. Multivariable regression analyses were conducted to identify characteristics associated with documented biomarker testing. Results: A total of 14,461 patients were identified: median age was 69.3 years, $52.3 \%$ were male, $14.6 \%$ were nonsmokers, and $64.7 \%$ had non-squamous histology. EGFR mutation testing rates were 35.5\% overall, with an increase in rates seen over time: $30.0 \%$ in 2012 to $44.0 \%$ in 2016 ( $\mathrm{p}<0.001)$. Anaplastic lymphoma kinase (ALK), c-ros oncogene 1 (ROS1), and programmed death-ligand 1 (PD-L1) mutation testing rates were $32.9 \%$, $5.7 \%$, and 5.7\%, respectively. More recent diagnosis year, non-squamous histology, larger practice size, and nonsmoking status were strongly associated with higher documented EGFR and ALK mutation testing rates. Conclusions: EGFR mutation testing rates steadily increased over time, but remained less than 50\%, with lower mutation testing rates reported for ALK, ROS1, and PD-L1, suggesting that opportunities exist to improve education on testing
\end{abstract}


for biomarkers in NSCLC.

\section{Keywords}

Non-Small-Cell Lung Cancer, NSCLC, Biomarkers, EGFR, Testing

\section{Introduction}

Lung cancer is the leading cause of cancer-related deaths in the USA. It is estimated that there will be 228,150 new cases and 142,670 deaths due to lung cancer in 2019 [1]. Approximately 85\% of lung cancers are non-small cell lung cancers (NSCLC), and non-squamous histologies are the most common. The most common non-squamous histologies are adenocarcinoma (approximately 40\%) and large cell (approximately 10\% - 15\%) [2]. Over the past few years, molecular targeted therapies have been emerging for the treatment of metastatic NSCLC with identified gene alterations. Actionable targets that can impact treatment selection include epidermal growth factor receptor (EGFR) mutations, anaplastic lymphoma kinase (ALK) rearrangements, c-ros oncogene 1 (ROS1) rearrangements, B-Raf proto-oncogene (BRAF) mutations, programmed death-ligand 1 (PD-L1) expression, and neurotrophic tyrosine kinase (NTRK) gene fusions [3] [4]. The use of targeted therapy is associated with improved outcomes in patients with advanced disease with identified gene alterations, and national guidelines recommend routine biomarker testing in patients with NSCLC so that those with gene alterations can receive treatment with effective targeted therapies [3] [4].

EGFR mutations are observed in approximately $40 \%$ and $20 \%$ of patients with NSCLC in Asian and non-Asian populations, respectively [5]. Mutations in exons 18 - 21 confer sensitivity to EGFR-tyrosine kinase inhibitors (TKIs) such as afatinib, erlotinib, and gefitinib. In patients with known EGFR mutations, frontline treatment with EGFR-TKIs is recommended. However, in approximately $50 \%$ of patients with EGFR mutation-positive NSCLC, a secondary mutation, EGFR T790M, develops, conferring resistance to first- and second-generation EGFR-TKI treatments [6]-[11]. Osimertinib, a third-generation, irreversible, oral EGFR-TKI, potently and selectively inhibits both EGFR sensitizing mutations and EGFR T790M, and has demonstrated efficacy in NSCLC central nervous system metastases [12]-[17]. Thus, continued biomarker testing in EGFR mutation-positive patients after progression on an EGFR-TKI may help identify patients who can continue to benefit from additional targeted therapies.

The primary objective of this study was to examine the rate of documented biomarker testing, including EGFR, EGFR T790M, ALK, ROS1, PD-L1, and BRAF, for patients with advanced NSCLC being treated in a US community oncology setting during the study. The secondary objective was to examine patient, disease, and provider factors associated with documented biomarker testing in patients with NSCLC in a real-world setting. 


\section{Materials and Methods}

\subsection{Study Design and Data Sources}

This was a retrospective observational study among patients who received care within a US Oncology Network clinic between January 1, 2012 and May 31, 2017. The US Oncology Network is affiliated with approximately 1400 physicians in more than 60 community oncology practices in over 450 sites of care across 25 states in the US. Patients were identified from practices using the iKnowMed (iKM) electronic health record (EHR) system. iKM is an oncology-specific EHR system that captures outpatient practice encounter history. Within the EHR, many data elements, including specific tumor biomarkers (EGFR, ALK, ROS1, PD-L1, and BRAF), are documented within structured data fields. Demographic and disease characteristic data including age, sex, race, smoking history, Eastern Cooperative Oncology Group performance status, histology, tumor biomarker status, and year of diagnosis were collected via programmatic queries of the iKM database. Eligible patients were at least 18 years of age at diagnosis of NSCLC, with Stage IV disease, and with at least two visits during the study period (January 2012 to May 2017). Patients enrolled in clinical trials at any time during the study period, and patients with other documented primary cancer diagnoses during the study period were excluded. The index date was defined as the date of Stage IV NSCLC diagnosis. The US Oncology Institutional Review Board approval was obtained for the study.

\subsection{Statistical Analysis}

Standard descriptive statistics were used for continuous and categorical study variables. Characteristics were calculated and compared using standard significance testing, such as chi-squared/Fisher's exact test (for categorical variables), and t-test/Mann-Whitney $U$ test/ANOVA/Kruskal-Wallis test (for the continuous variables). Patients were examined overall and by histology. Rates of documented biomarker testing were calculated, overall and by year. All patients who met the eligibility criteria and had results from documented biomarker testing were included. The actual testing date for each patient was also collected. Multivariable stepwise logistic regression analysis was conducted to identify characteristics associated with documented biomarker testing. The stepwise model building process used a type $3 \mathrm{p}$-value for entry of 0.20 and type $3 \mathrm{p}$-value for retention of 0.10 . Odds ratios and $95 \%$ confidence intervals (CIs) were reported. For any significance testing, an alpha of 0.05 was used unless otherwise stated or requested. The analyses were conducted using SAS ${ }^{\circledast}$ (SAS Institute Inc., Version 9.4, Cary, NC, US).

\section{Results}

\subsection{Patient Characteristics}

There were 14,461 patients with advanced NSCLC meeting eligibility during the 5 -year study period. Table 1 describes the demographic and clinical characteristics 
Table 1. Demographic and clinical characteristics among patients with NSCLC, overall and by histologic type.

\begin{tabular}{|c|c|c|c|c|c|}
\hline & $\begin{array}{c}\text { Overall } \\
(\mathrm{N}=14,461)\end{array}$ & $\begin{array}{l}\text { Non-Squamous } \\
\qquad(\mathrm{N}=9,359)\end{array}$ & $\begin{array}{l}\text { Squamous } \\
(\mathrm{N}=2,527)\end{array}$ & $\begin{array}{l}\text { Unspecified Non-Small } \\
\text { Cell Cancer }(\mathrm{N}=471)\end{array}$ & $\begin{array}{l}\text { Not Documented } \\
\qquad(\mathrm{N}=2,104)\end{array}$ \\
\hline \multicolumn{6}{|c|}{ Age, years } \\
\hline Median (Min, Max) & $69.3(21.5,90+)$ & $68.9(21.5,90+)$ & $71.0(28.4,90+)$ & $68.6(34.9,90+)$ & $69.5(30.3,90+)$ \\
\hline \multicolumn{6}{|c|}{ Sex, n (\%) } \\
\hline Female & $6901(47.7)$ & $4694(50.2)$ & $967(38.3)$ & $215(45.6)$ & $1025(48.7)$ \\
\hline Male & $7560(52.3)$ & $4665(49.8)$ & $1560(61.7)$ & $256(54.4)$ & $1079(51.3)$ \\
\hline \multicolumn{6}{|c|}{ Race, N (\%) } \\
\hline White & $10,949(75.7)$ & $7052(75.4)$ & $2006(79.4)$ & $367(77.9)$ & $1524(72.4)$ \\
\hline Black & $1271(8.8)$ & $804(8.6)$ & $213(8.4)$ & $48(10.2)$ & $206(9.8)$ \\
\hline Asian & $372(2.6)$ & $284(3.0)$ & $31(1.2)$ & $6(1.3)$ & $51(2.4)$ \\
\hline Other & $159(1.1)$ & $103(1.1)$ & $28(1.1)$ & $7(1.5)$ & $21(1.0)$ \\
\hline Not documented & $1710(11.8)$ & $1116(11.9)$ & $249(9.9)$ & $43(9.1)$ & $302(14.4)$ \\
\hline \multicolumn{6}{|c|}{ Smoking History, N (\%) } \\
\hline Former & $8652(59.8)$ & $5541(59.2)$ & $1648(65.2)$ & $298(63.3)$ & $1165(55.4)$ \\
\hline Current & $2619(18.1)$ & $1559(16.7)$ & $553(21.9)$ & $95(20.2)$ & $412(19.6)$ \\
\hline Never & $2115(14.6)$ & $1637(17.5)$ & $156(6.2)$ & $45(9.6)$ & $277(13.2)$ \\
\hline Not documented & $1075(7.4)$ & $622(6.6)$ & $170(6.7)$ & $33(7.0)$ & $250(11.9)$ \\
\hline \multicolumn{6}{|c|}{ ECOG Performance Status at Index, N (\%) } \\
\hline 0 & $477(3.3)$ & $356(3.8)$ & $61(2.4)$ & $10(2.1)$ & $50(2.4)$ \\
\hline 1 & $6277(43.4)$ & $4254(45.5)$ & $1083(42.9)$ & $197(41.8)$ & $743(35.3)$ \\
\hline 2 & $4009(27.7)$ & $2598(27.8)$ & $772(30.6)$ & $146(31.0)$ & $493(23.4)$ \\
\hline $3+$ & $1236(8.5)$ & $802(8.6)$ & $218(8.6)$ & $49(10.4)$ & $167(7.9)$ \\
\hline Not documented & $2462(17.0)$ & $1349(14.4)$ & $393(15.6)$ & $69(14.7)$ & $651(30.9)$ \\
\hline \multicolumn{6}{|c|}{ Practice Patient Volume (NSCLC Patients Treated/Year), N (\%) } \\
\hline$<50$ & $4519(31.3)$ & 2957 (31.6) & $804(31.8)$ & $144(30.6)$ & $614(29.2)$ \\
\hline $50-99$ & $5518(38.2)$ & $3638(38.9)$ & $1002(39.7)$ & $162(34.4)$ & $716(34.0)$ \\
\hline $100-149$ & $1135(7.8)$ & $742(7.9)$ & $196(7.8)$ & $42(8.9)$ & $155(7.4)$ \\
\hline $150+$ & $903(6.2)$ & $622(6.6)$ & $122(4.8)$ & $30(6.4)$ & $129(6.1)$ \\
\hline Not documented & $2386(16.5)$ & $1400(15.0)$ & $403(15.9)$ & $93(19.7)$ & $490(23.3)$ \\
\hline \multicolumn{6}{|c|}{ Practice Physician Size, N (\%) } \\
\hline Small (0 - 5 physicians) & $3124(21.6)$ & $1983(21.2)$ & $617(24.4)$ & $113(24.0)$ & $411(19.5)$ \\
\hline Medium (6 - 10 physicians) & $6360(44.0)$ & $4222(45.1)$ & $1092(43.2)$ & $185(39.3)$ & $861(40.9)$ \\
\hline Large ( $>10$ physicians) & $2296(15.9)$ & $1590(17.0)$ & $346(13.7)$ & $70(14.9)$ & $290(13.8)$ \\
\hline Not documented & $2681(18.5)$ & $1564(16.7)$ & $472(18.7)$ & $103(21.9)$ & $542(25.8)$ \\
\hline \multicolumn{6}{|c|}{ Practice Region, N (\%) } \\
\hline South & $8852(61.2)$ & $5589(59.7)$ & $1654(65.5)$ & $275(58.4)$ & $1334(63.4)$ \\
\hline West & $3088(21.4)$ & $2033(21.7)$ & $499(19.7)$ & $105(22.3)$ & $451(21.4)$ \\
\hline Midwest & $1513(10.5)$ & $1043(11.1)$ & $219(8.7)$ & $48(10.2)$ & $203(9.6)$ \\
\hline Northeast & $1008(7.0)$ & $694(7.4)$ & $155(6.1)$ & $43(9.1)$ & $116(5.5)$ \\
\hline
\end{tabular}

ECOG, Eastern Cooperative Oncology Group; max, maximum; min, minimum; NSCLC, non-small cell lung cancer. 
of the patients overall, and by histologic subtype. Most patients (64.7\%) had non-squamous histology, consisting of $59.8 \%$ of patients $(n=8644)$ with adenocarcinoma. The median age at diagnosis was 69.3 years. Most patients were treated at medium-sized practices with six to ten physicians.

\subsection{Testing Patterns}

Less than half of patients overall and in all histology groups had documentation of EGFR testing (Table 2). The lowest proportion of patients tested for a EGFR

Table 2. Documented mutation testing and test results among patients with NSCLC for full study period, overall and by histologic type.

\begin{tabular}{|c|c|c|c|c|c|}
\hline & $\begin{array}{c}\text { Overall } \\
(\mathrm{N}=14,461)\end{array}$ & $\begin{array}{l}\text { Non-Squamous } \\
\quad(\mathrm{N}=9,359)\end{array}$ & $\begin{array}{l}\text { Squamous } \\
(\mathrm{N}=2,527)\end{array}$ & $\begin{array}{l}\text { Unspecified Non-Small } \\
\text { Cell Cancer }(\mathrm{N}=471)\end{array}$ & $\begin{array}{l}\text { Not Documented } \\
\qquad(\mathrm{N}=2,104)\end{array}$ \\
\hline \multicolumn{6}{|c|}{ EGFR Status, N (\%) } \\
\hline Patients tested & $5132(35.5)$ & $4456(47.6)$ & $358(14.2)$ & $120(25.5)$ & $198(9.4)$ \\
\hline Negative & 4098 (79.9) & $3500(78.5)$ & $330(92.2)$ & $111(92.5)$ & $157(79.3)$ \\
\hline Positive $^{1}$ & $1016(19.8)$ & $938(21.1)$ & $28(7.8)$ & $9(7.5)$ & $41(20.7)$ \\
\hline Tested, but result unknown & $18(0.4)$ & $18(0.4)$ & 0 & 0 & 0 \\
\hline No documented testing & $9329(64.5)$ & $4903(52.4)$ & $2169(85.8)$ & $351(74.5)$ & $1906(90.6)$ \\
\hline \multicolumn{6}{|c|}{ ALK Status, N (\%) } \\
\hline Patients tested & $4752(32.9)$ & $4121(44.0)$ & $337(13.3)$ & $119(25.3)$ & $175(8.3)$ \\
\hline Negative & $4448(93.6)$ & $3849(93.4)$ & $322(95.5)$ & $111(93.3)$ & $166(94.9)$ \\
\hline Positive & $204(4.3)$ & $189(4.6)$ & $8(2.4)$ & $1(0.8)$ & $6(3.4)$ \\
\hline Tested, but result unknown & $100(2.1)$ & $83(2.0)$ & $7(2.1)$ & $7(5.9)$ & $3(1.7)$ \\
\hline No documented testing & $9709(67.1)$ & $5238(56.0)$ & $2190(86.7)$ & $352(74.7)$ & $1929(91.7)$ \\
\hline \multicolumn{6}{|c|}{ ROS1 Status, N (\%) } \\
\hline Patients tested & $820(5.7)$ & $712(7.6)$ & $91(3.6)$ & $15(3.2)$ & $2(0.1)$ \\
\hline Negative & 797 (97.2) & $690(96.9)$ & $91(100.0)$ & $14(93.3)$ & $2(100.0)$ \\
\hline Positive & $23(2.8)$ & $22(3.1)$ & 0 & $1(6.7)$ & 0 \\
\hline No documented testing & $13,641(94.3)$ & $8647(92.4)$ & $2436(96.4)$ & $456(96.8)$ & $2102(99.9)$ \\
\hline \multicolumn{6}{|c|}{ PD-L1 Status, N (\%) } \\
\hline Patients tested & $831(5.7)$ & $673(7.2)$ & $135(5.3)$ & $19(4.0)$ & $4(0.2)$ \\
\hline Negative & $487(58.6)$ & $402(59.7)$ & $78(57.8)$ & $7(36.8)$ & 0 \\
\hline Positive & $344(41.4)$ & $271(40.3)$ & $57(42.2)$ & $12(63.2)$ & $4(100.0)$ \\
\hline No documented testing & $13,630(94.3)$ & $8686(92.8)$ & $2392(94.7)$ & $452(96.0)$ & $2100(99.8)$ \\
\hline \multicolumn{6}{|c|}{ BRAF Status, N (\%) } \\
\hline Patients tested & $16(0.1)$ & $15(0.2)$ & 0 & $1(0.2)$ & 0 \\
\hline Negative & $13(81.3)$ & $13(86.7)$ & 0 & 0 & 0 \\
\hline Positive & $3(18.8)$ & $2(13.3)$ & 0 & $1(100.0)$ & 0 \\
\hline No documented testing & 14,445 (99.9) & $9344(99.8)$ & $2527(100.0)$ & $470(99.8)$ & $2104(100.0)$ \\
\hline
\end{tabular}

The denominator for the proportion of patients with negative/positive/result unknown mutation test results is the number of patients tested. Percentages may not add up to 100 due to rounding. ${ }^{1}$ Information on whether mutation was sensitizing was provided if available in iKM. Positive results include EGFR-TKI sensitizing mutation $(+) \mathrm{n}=511$; EGFR-TKI non-sensitizing mutation $(+) \mathrm{n}=67$; T790M $\mathrm{n}=39$. ALK, anaplastic lymphoma kinase; BRAF, B-Raf proto-oncogene, EGFR, epidermal growth factor receptor; PD-L1, programmed death-ligand 1; NSCLC, non-small cell lung cancer; ROS1, c-ros oncogene 1 . 
mutation were those with squamous cell carcinoma (14.2\%) or no documented histology (9.4\%). Of those with documentation of EGFR mutation testing, $19.8 \%$ were positive, and those with non-squamous histology had the highest proportions of EGFR mutation positive patients (21.1\%). Of those with and without documented EGFR mutation testing, 7.0\% in the overall group were positive.

EGFR testing rates from diagnosis to the end of the study period were $35.5 \%$ overall, with an increase in rates observed over time: $30.0 \%$ in 2012 to $44.0 \%$ in 2016 ( $\mathrm{p}<0.001$ ) (Figure 1(a), Figure 1(b)). Data for 2017 was only available for the partial year. During the 5-year study period, the proportion of patients tested for EGFR mutations was at its highest point in 2016. Among these patients tested in 2016, 19.4\% tested positive (data not shown). Data on testing for

Patients tested ${ }^{1}$ and patients diagnosed from 2012- 2017

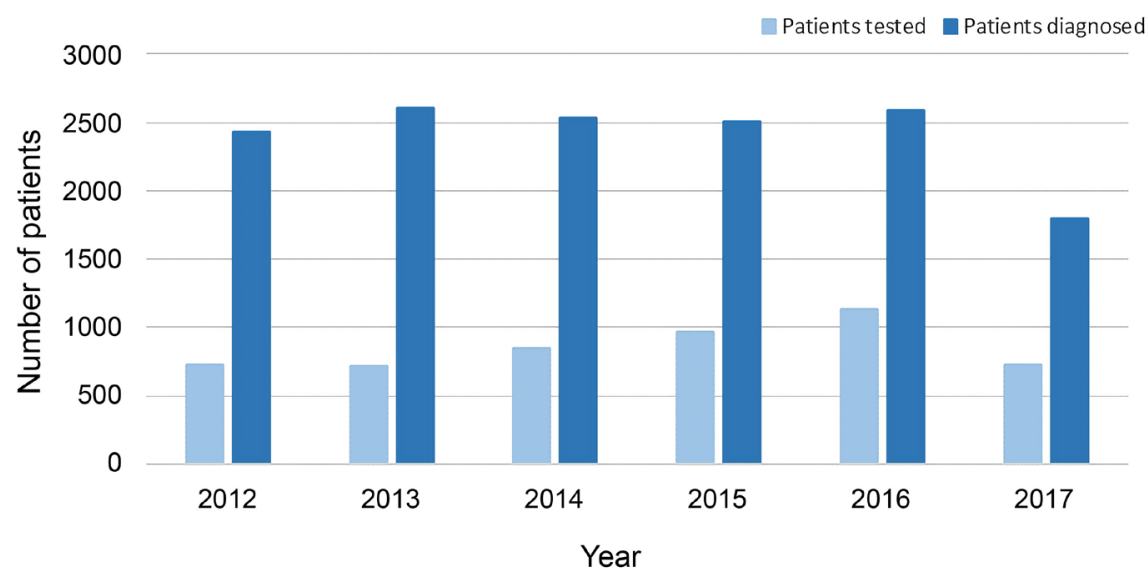

(a)

\section{Patients with documented testing (\%)}

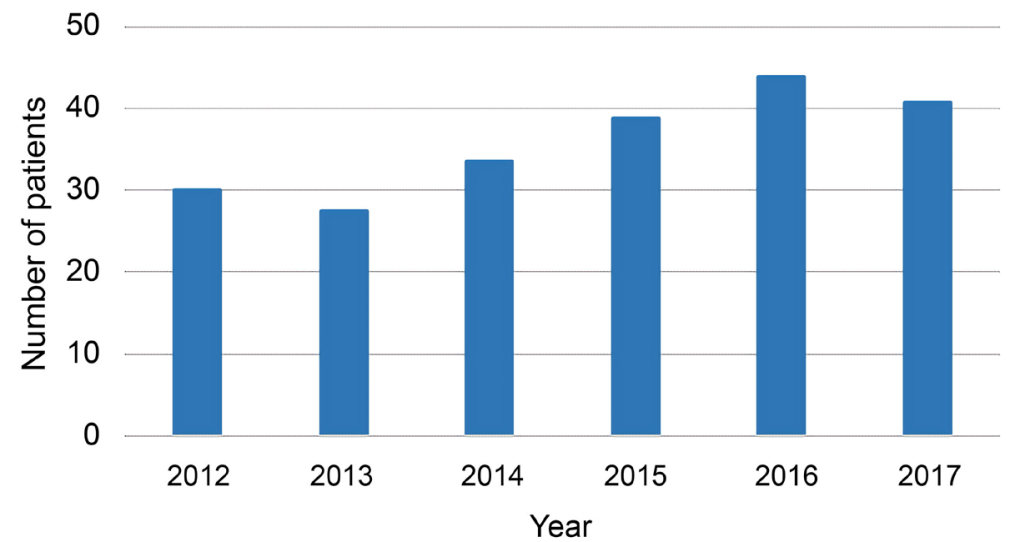

(b)

Figure 1. (a) Patients diagnosed and patients tested for EGFR mutations by year ${ }^{\star} .{ }^{\star}$ Data for 2017 was a partial year; (b) proportion of patients with documented EGFR mutation testing by year*. Data are as documented in the EHR. Lack of documentation does not necessarily confirm that the patient was not tested. ${ }^{\star}$ Data for 2017 was a partial year. EGFR, epidermal growth factor receptor; EHR, electronic health record. 
the EGFR T790M mutation specifically in the EHR were available for 2016 and 2017 only (data not shown). Of the known EGFR mutation-positive patients, 9.5\% and 10.8\% were tested for EGFR T790M in 2016 and 2017 respectively.

Similarly, 32.9\% of patients overall had documentation of ALK status testing. Of those with documented mutation testing, few had an ALK-positive status (4.3\% overall).

ROS1 or PD-L1 status testing was documented during the study period in $5.7 \%$ of patients. Among those who did, most were negative for a ROS1 mutation (97.2\%) and PD-L1 expression (58.6\%). BRAF testing was conducted in $0.1 \%$ of patients. Testing would not have been performed for these biomarkers during the earlier years of this study when these tests were not yet available or actionable.

\subsection{Predictors of Testing}

In a multivariate logistic regression analysis, several parameters were strongly associated with higher documented EGFR mutation testing rates (Table 3). Patients with a non-squamous histology (including adenocarcinoma and bronchioalveolar) were more likely to have been tested for EGFR compared with patients with a squamous histology $(\mathrm{p}<0.0001)$. The likelihood of being tested increased with more recent diagnoses, increasing by 2.8-fold from 2013 to 2017 ( $\mathrm{p}<$ $0.0001)$. A larger practice size $(\mathrm{p}=0.0097)$ and volume $(<50$ patients versus $\geq 50$ patients; $\mathrm{p}<0.0001)$ were also associated with higher documented testing rates, as were nonsmoking status (former or never) compared with current smokers $(\mathrm{p}<0.0001)$, being female $(\mathrm{p}=0.005)$, and practice region $(\mathrm{p}=0.013)$. Race had no effect on documented EGFR testing rates.

A similar analysis was performed for documented ALK mutation testing rates with comparable results observed (Table 4). Factors associated with having documented ALK testing included adenocarcinoma and bronchioalveolar histologies

Table 3. Multivariable model examining associations between patient and practice level characteristics and documented EGFR mutation testing.

\begin{tabular}{|c|c|c|c|c|}
\hline & Characteristic & Total & Odds Ratio ( $95 \% \mathrm{CI}$ ) & p-Value \\
\hline \multirow[t]{3}{*}{ Age } & Per year increase & 8423 & $0.99(0.99-0.99)$ & $<0.0001$ \\
\hline & Squamous (reference) & 1721 & . & \\
\hline & Adenocarcinoma & 5930 & $5.05(4.38-5.82)$ & \\
\hline \multirow[t]{4}{*}{ Histology } & Other & 523 & $1.52(1.19-1.94)$ & $<0.0001$ \\
\hline & Adenosquamous & 178 & $3.55(2.53-4.99)$ & \\
\hline & Bronchioloalveolar carcinoma & 71 & $5.56(3.36-9.22)$ & \\
\hline & South (reference) & 5288 & . & \\
\hline \multirow{3}{*}{ Practice Region } & West & 1767 & $0.95(0.84-1.07)$ & \\
\hline & Midwest & 880 & $1.33(1.13-1.57)$ & 0.0013 \\
\hline & Northeast & 488 & $0.93(0.75-1.15)$ & \\
\hline
\end{tabular}




\section{Continued}

\begin{tabular}{|c|c|c|c|c|}
\hline & Small (reference) & 2327 & . & \\
\hline \multirow[t]{3}{*}{ Practice size } & Medium & 4465 & $1.135(1.00-1.29)$ & \multirow[t]{2}{*}{0.0097} \\
\hline & Large & 1631 & $1.33(1.11-1.60)$ & \\
\hline & $<50$ (reference) & 2994 & . & \multirow{4}{*}{$<0.0001$} \\
\hline \multirow{3}{*}{ Practice Volume } & $50-99$ & 4007 & $0.80(0.71-0.91)$ & \\
\hline & $100-149$ & 824 & $0.94(0.77-1.15)$ & \\
\hline & $150+$ & 598 & $0.60(0.41-0.75)$ & \\
\hline \multirow{3}{*}{ Smoking Status } & Current (reference) & 1631 & . & \multirow{3}{*}{$<0.0001$} \\
\hline & Former & 5439 & $1.35(1.19-1.53)$ & \\
\hline & Never & 1353 & $2.00(1.70-2.36)$ & \\
\hline \multirow{2}{*}{ Sex } & Male (reference) & 4402 & . & \multirow{2}{*}{0.0005} \\
\hline & Female & 4021 & $1.19(1.08-1.30)$ & \\
\hline \multirow{4}{*}{ Race } & White (reference) & 7345 & . & \multirow{4}{*}{0.0769} \\
\hline & Black & 700 & $0.97(0.82-1.16)$ & \\
\hline & Asian & 266 & $1.30(0.99-1.72)$ & \\
\hline & Other & 112 & $1.47(0.97-2.22)$ & \\
\hline \multirow{6}{*}{ Year of Diagnosis } & 2012 (reference) & 952 & . & \multirow{6}{*}{$<0.0001$} \\
\hline & 2013 & 1403 & $1.34(1.11-1.63)$ & \\
\hline & 2014 & 1479 & $1.86(1.54-2.24)$ & \\
\hline & 2015 & 1579 & $2.56(2.13-3.08)$ & \\
\hline & 2016 & 1663 & $3.10(2.57-3.72)$ & \\
\hline & 2017 & 1347 & $3.77(3.11-4.56)$ & \\
\hline
\end{tabular}

Table 4. Multivariable model examining associations between patient and practice level characteristics and documented ALK mutation testing.

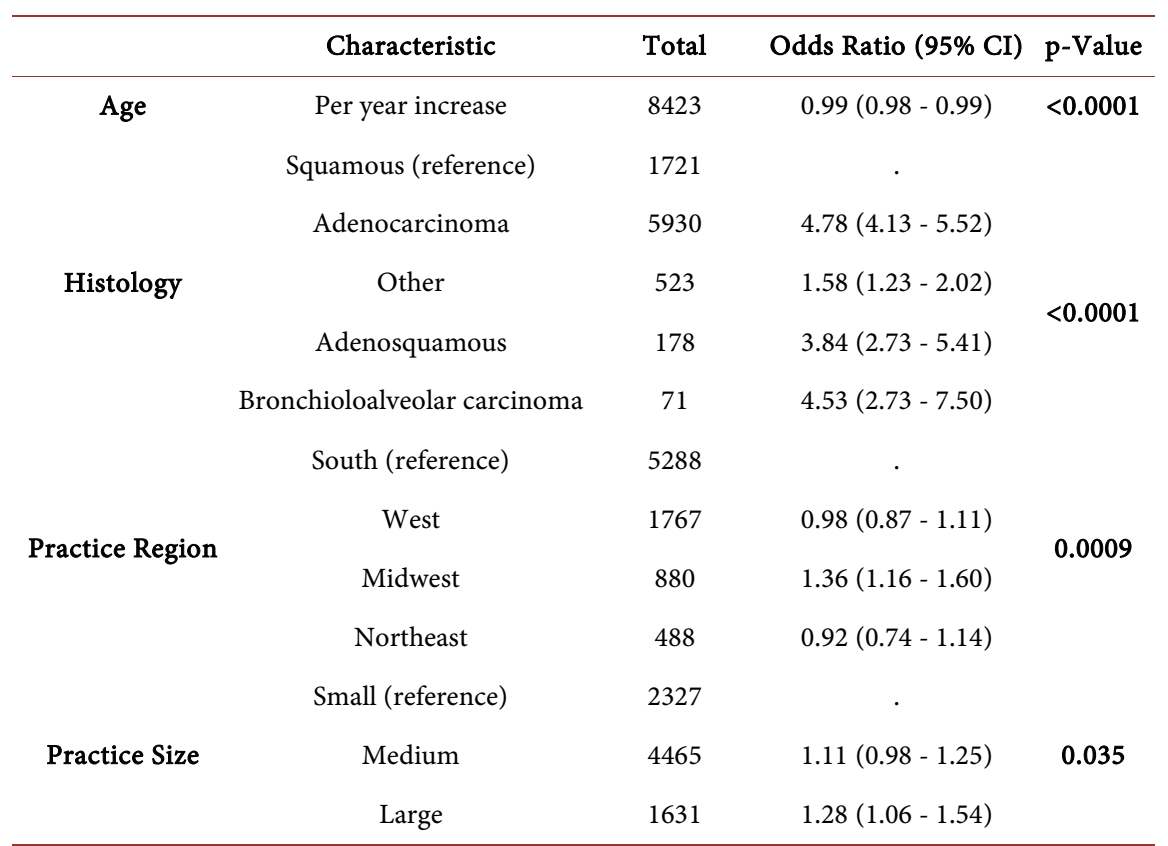




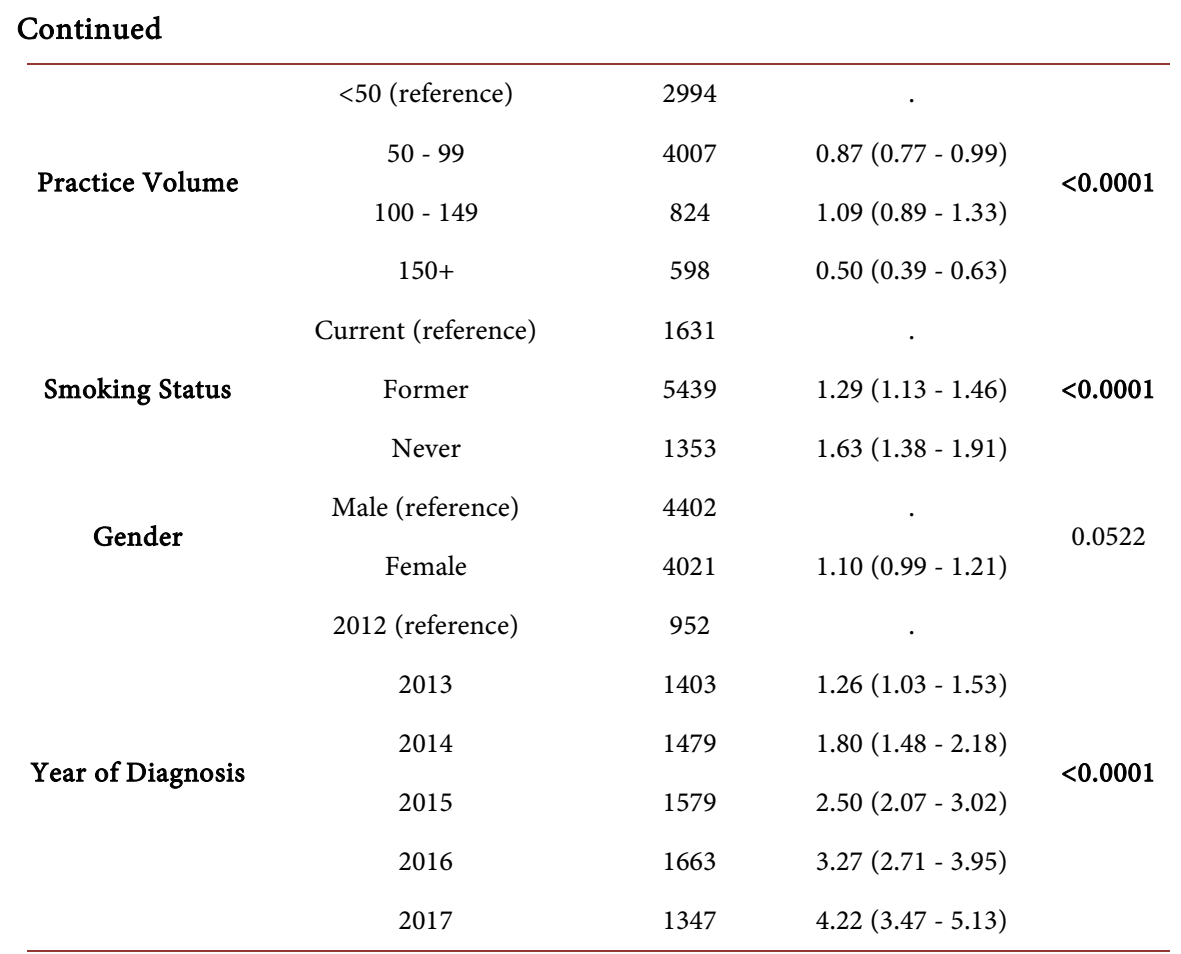

( $\mathrm{p}<0.0001)$, nonsmoking status $(\mathrm{p}<0.0001)$, and practice size $(\mathrm{p}=0.035)$, volume ( $p<0.0001)$, and region $(\mathrm{p}=0.0009)$. The likelihood of testing again increased with more recent diagnoses: those diagnosed in 2017 were 3.3 times more likely to have had ALK testing as those diagnosed in 2013 ( $\mathrm{p}<0.0001$ ). Unlike EGFR mutation testing rates, gender had no significant association with documented ALK mutation testing rates.

Testing rates for other biomarkers were low, therefore a predictive modelling was not performed.

\section{Discussion}

Although clinical practice guidelines recommend biomarker testing in all NSCLC patients, little is known about actual testing rates in the real-world setting, particularly in community settings. In our study, testing rates for EGFR mutations specifically were $35 \%$ overall, with an increase observed over time from $30 \%$ in 2012 to $41 \%$ in 2016. The testing rate for ALK rearrangements was $33 \%$ overall.

It was not unreasonable that testing rates for the other actionable biomarkers in our study were low, given the timeframe of this study in relation to the approval of other targeted therapies for ROS1, BRAF, and PD-L1. This study evaluated testing in the years between 2012 and 2017. PD-L1 inhibitors for the treatment of NSCLC became available in 2015, and the recommendations for PD-L1 testing have changed over time. Similarly, targeted therapy for the treatment of ROS1-positive NSCLC and BRAF-positive NSCLC became available in 2016 and 2017, respectively. Also, NTRK gene fusion testing was not included in 
this study as this test was added to the guidelines after the study was completed. However, biomarker testing remains important in the management of advanced NSCLC to identify patients eligible for targeted therapies which may improve their outcomes.

In one study by McKeage et al., the real-world uptake of EGFR mutation testing was assessed during the implementation of updated testing guidelines in a registry cohort from New Zealand. From 2010 to 2014, 1857 non-squamous NSCLC patients were identified as being eligible for EGFR testing [18]. Testing occurred in $27 \%$ of patients. Testing rates increased during the study period from $<5 \%$ to $67 \%$ of patients ( $\mathrm{p}<0.0001$ ). It was demonstrated that testing for EGFR mutations was associated with increased survival (adjusted hazard ratio = 0.76 [95\% CI 0.65 - 0.89]; log-rank $\mathrm{p}<0.0001$ ), which was thought to be driven by longer survival in the EGFR mutation-positive patients receiving targeted therapy. This reinforces the need for testing in the NSCLC population to determine which patients are candidates for targeted therapies.

Reasons for not performing EGFR mutation testing in the McKeage study included a lack of availability of specimens. This may likely have also played a role in the present study. Surgery- or biopsy-obtained tumor tissue may not always be available, and testing may be a challenge if samples are small. Additionally, performing invasive procedures may pose a risk for some patients. It has been reported that $20 \%-30 \%$ of patients are not able to provide tumor samples at diagnosis [19]. Therefore, other methods such as using circulating free tumorderived DNA from plasma have been tested. Additionally, the use of large-scale sequencing strategies, such as next-generation sequencing, allows for the detection of multiple molecular targets simultaneously. Targeted educational interventions are needed regarding how and when biomarker testing should be performed to optimize and support personalized treatment for NSCLC. We observed that non-squamous histology, larger practice size, and nonsmoking status were strongly associated with higher documented EGFR mutation testing rates in our study. This mutation is often found in nonsmoking women from East Asian decent with non-squamous histology [2]. Increased knowledge and education regarding patient and clinical characteristics associated with specific gene alterations may also improve testing rates.

One study reported an increase in EGFR testing after implementation of reflex testing, defined as a request for EGFR testing by the pathologist at the time of non-squamous NSCLC diagnosis [20]. From 2010 to 2014, of 2214 patients from seven centers in Canada that had EGFR testing, 1330 patients were tested before implementation of reflex testing and 884 patients were tested after. During this time, the proportion of pathologists requesting mutation testing increased from $4 \%$ to $53 \%$, whereas the proportion of mutation testing requested by medical oncologists decreased from $95 \%$ to $46 \%$ ( $p<0.001)$. There was a significant increase in the number of patients tested per center per month $(\mathrm{p}<0.001)$. Thus, it was observed that reflex testing could help increase awareness and reduce bar- 
riers to testing.

Testing data for EGFR T790M in this study was only available for 2016 and 2017, reflecting the approval timing for the EGFR T790M mutation test. The testing rate overall was low, with less than $10 \%$ of known EGFR mutation-positive patients receiving testing for EGFR T790M during the study period. With the recent availability of additional targeted therapy options, including third-generation EGFR-TKIs such as osimertinib, in patients who develop resistance following first- and second-generation EGFR-TKIs, increased awareness of new treatment options will help support ongoing personalized therapy in advanced NSCLC.

In our study, $33 \%$ of patients received ALK status testing. Lower testing rates have been reported in other studies with newly diagnosed advanced NSCLC; ALK status was tested in $18 \%$ of patients in the international PIvOTAL observational study $(\mathrm{n}=1440)$ [21] and in $17 \%$ of patients in a retrospective Japanese study $(\mathrm{n}=175)$ [22]. The other biomarkers tested in this study have more recently been added to the advanced NSCLC guidelines so there are few studies analyzing testing rates to compare with our data. The recommended timing for when to conduct biomarker testing in NSCLC has also evolved over the years. At the time that this study was performed, biomarker testing was recommended for all patients with Stage IV NSCLC. Improved understanding and better dissemination of updates to guideline recommendations for testing timing and methods may also improve testing rates overall.

Limitations of this study include the retrospective observational nature of the study with data extracted from a database, and the potential for under-reported testing. Availability of biomarker testing required that results were directly entered into the specific structured EHR fields. Results available through scanned documents or progress notes may not have been entered into the specific fields in the EHR, and therefore reasons for not testing could not be investigated. The low rate of EGFR mutation testing (35\%) was likely a consequence of this structured data extraction methodology, as well as a general lack of documentation. Also, biomarker testing rates were captured when some therapies were not approved, thereby impacting what biomarkers were actionable for documentation as structured data in the EHR. Furthermore, at the time of the study, osimertinib was not approved as first-line treatment for EGFR mutated NSCLC patients which may have an impact on translating these data to current practice. Strengths of this study include the large sample size of over 14,000 advanced NSCLC patients evaluated in community oncology practices across the US, reflecting real-world community practice and testing patterns.

\section{Conclusion}

Between 2012 and 2016, EGFR mutation testing rates steadily increased over time in patients with Stage IV NSCLC but remained less than $50 \%$. Testing rates for ALK, ROS1, PD-L1, and BRAF were lower, ranging from $<1 \%$ to $33 \%$. Our data, therefore, suggest that opportunities exist to improve biomarker testing 
uptake and education in advanced NSCLC in the real world. The reasons for not testing should be identified to understand actions needed to improve biomarker testing rates.

\section{Acknowledgements}

The authors would like to acknowledge Robert Harrison, $\mathrm{PhD}$, of iMed Comms, Macclesfield, UK, an Ashfield Company, part of UDG Healthcare plc, for technical editing support that was funded by AstraZeneca in accordance with Good Publications Practice (GPP3) guidelines (http://www.ismpp.org/gpp3).

\section{Conflicts of Interest}

Eric Nadler reports a consulting or advisory role for Merck, and participation in speakers' bureau for Merck, Genentech, and AstraZeneca. Janet Espirito and Jamyia Clark are McKesson employees and shareholders. Melissa Pavilack is an AstraZeneca employee. Ancilla Fernandes is an AstraZeneca employee and shareholder. All authors critically reviewed the manuscript and approved the final version for submission.

\section{References}

[1] Siegel, R.L., Miller, K.D. and Jemal, A. (2018) Cancer Statistics, 2018. CA: A Cancer Journal for Clinicians, 68, 7-30. https://doi.org/10.3322/caac.21442

[2] Zappa, C. and Mousa, S.A. (2016) Non-Small Cell Lung Cancer: Current Treatment and Future Advances. Translational Lung Cancer Research, 5, 288-300. https://doi.org/10.21037/tlcr.2016.06.07

[3] Hanna, N., Johnson, D., Temin, S., et al. (2017) Systemic Therapy for Stage IV NonSmall-Cell Lung Cancer: American Society of Clinical Oncology Clinical Practice Guideline Update. Journal of Clinical Oncology, 35, 3484-3515. https://doi.org/10.1200/JCO.2017.74.6065

[4] Planchard, D., Popat, S., Kerr, K., et al. (2018) Metastatic Non-Small Cell Lung Cancer: ESMO Clinical Practice Guidelines for Diagnosis, Treatment and FollowUp. Annals of Oncology, 29, iv192-iv237. https://doi.org/10.1093/annonc/mdy275

[5] Zhang, Y.L., Yuan, J.Q., Wang, K.F., et al. (2016) The Prevalence of EGFR Mutation in Patients with Non-Small Cell Lung Cancer: A Systematic Review and Meta-Analysis. Oncotarget, 7, 78985-78993. https://doi.org/10.18632/oncotarget.12587

[6] Jenkins, S., Yang, J.C., Ramalingam, S.S., et al. (2017) Plasma ctDNA Analysis for Detection of the EGFR T790M Mutation in Patients with Advanced Non-Small Cell Lung Cancer. Journal of Thoracic Oncology, 12, 1061-1070. https://doi.org/10.1016/j.jtho.2017.04.003

[7] Arcila, M.E., Oxnard, G.R., Nafa, K., et al. (2011) Rebiopsy of Lung Cancer Patients with Acquired Resistance to EGFR Inhibitors and Enhanced Detection of the T790M Mutation Using a Locked Nucleic Acid-Based Assay. Clinical Cancer Research, 17, 1169-1180. https://doi.org/10.1158/1078-0432.CCR-10-2277

[8] Sun, J.M., Ahn, M.J., Choi, Y.L., Ahn, J.S. and Park, K. (2013) Clinical Implications of T790M Mutation in Patients with Acquired Resistance to EGFR Tyrosine Kinase Inhibitors. Lung Cancer, 82, 294-298. https://doi.org/10.1016/j.lungcan.2013.08.023 
[9] Kuiper, J.L., Heideman, D.A., Thunnissen, E., et al. (2014) Incidence of T790M Mutation in (Sequential) Rebiopsies in EGFR-Mutated NSCLC-Patients. Lung Cancer, 85, 19-24. https://doi.org/10.1016/j.lungcan.2014.03.016

[10] Li, W., Ren, S., Li, J., et al. (2014) T790M Mutation Is Associated with Better Efficacy of Treatment beyond Progression with EGFR-TKI in Advanced NSCLC Patients. Lung Cancer, 84, 295-300. https://doi.org/10.1016/j.lungcan.2014.03.011

[11] Akamatsu, H.A.D., John, T., Su, W.-C., Lee, J.-S., Chang, G.-C., Huang, X., Jenkins, S., Dearden, S. and Wu, Y.-L. (2017) 421P EGFR Mutation Analysis for Prospective Patient (pt) Selection in AURA3 Phase III Trial of Osimertinib vs Platinum-Pemetrexed (plt-pem) in pts with EGFR T790M Positive Advanced Non-Small Cell Lung Cancer (NSCLC). Annals of Oncology, 28, x124-x143.

https://doi.org/10.1093/annonc/mdx671.010

[12] Jänne, P.A., Yang, J.C., Kim, D.W., et al. (2015) AZD9291 in EGFR Inhibitor-Resistant Non-Small-Cell Lung Cancer. The New England Journal of Medicine, 372, 1689-1699. https://doi.org/10.1056/NEJMoa1411817

[13] Cross, D.A., Ashton, S.E., Ghiorghiu, S., et al. (2014) AZD9291, an Irreversible EGFR TKI, Overcomes T790M-Mediated Resistance to EGFR Inhibitors in Lung Cancer. Cancer Discovery, 4, 1046-1061. https://doi.org/10.1158/2159-8290.CD-14-0337

[14] Mok, T.S., Wu, Y.-L., Ahn, M.-J., et al. (2017) Osimertinib or Platinum-Pemetrexed in EGFR T790M-Positive Lung Cancer. The New England Journal of Medicine, 376, 629-640. https://doi.org/10.1056/NEJMoa1612674

[15] Soria, J.C., Ohe, Y., Vansteenkiste, J., et al. (2018) Osimertinib in Untreated EGFRMutated Advanced Non-Small-Cell Lung Cancer. The New England Journal of Medicine, 378, 113-125. https://doi.org/10.1056/NEJMoa1713137

[16] Wu, Y.L., Ahn, M.J., Garassino, M.C., et al. (2018) CNS Efficacy of Osimertinib in Patients with T790M-Positive Advanced Non-Small-Cell Lung Cancer: Data from a Randomized Phase III Trial (AURA3). Journal of Clinical Oncology, 36, 2702-2709. https://doi.org/10.1200/JCO.2018.77.9363

[17] Reungwetwattana, T., Nakagawa, K., Cho, B.C., et al. (2018) CNS Response to Osimertinib versus Standard Epidermal Growth Factor Receptor Tyrosine Kinase Inhibitors in Patients with Untreated EGFR-Mutated Advanced Non-Small-Cell Lung Cancer. Journal of Clinical Oncology, 36, 3290-3297. https://doi.org/10.1200/JCO.2018.78.3118

[18] McKeage, M., Elwood, M., Tin, S., et al. (2017) EGFR Mutation Testing of NonSquamous NSCLC: Impact and Uptake during Implementation of Testing Guidelines in a Population-Based Registry Cohort from Northern New Zealand. Targeted Oncology, 12, 663-675. https://doi.org/10.1007/s11523-017-0515-4

[19] Herbreteau, G., Vallee, A., Charpentier, S., Normanno, N., Hofman, P. and Denis, M.G. (2019) Circulating Free Tumor DNA in Non-Small Cell Lung Cancer (NSCLC): Clinical Application and Future Perspectives. Journal of Thoracic Disease, 11, S113-s126. https://doi.org/10.21037/jtd.2018.12.18

[20] Cheema, P.K., Raphael, S., El-Maraghi, R., et al. (2017) Rate of EGFR Mutation Testing for Patients with Nonsquamous Non-Small-Cell Lung Cancer with Implementation of Reflex Testing by Pathologists. Current Oncology, 24, 16-22.

https://doi.org/10.3747/co.24.3266

[21] Lee, D.H., Tsao, M.-S., Kambartel, K.-O., et al. (2018) Molecular Testing and Treatment Patterns for Patients with Advanced Non-Small Cell Lung Cancer: PIvOTAL Observational Study. PLoS ONE, 13, e0202865.

https://doi.org/10.1371/journal.pone.0202865 
[22] Isobe, H., Mori, K., Minato, K., et al. (2017) Real-World Practice Patterns for Patients with Advanced Non-Small Cell Lung Cancer: Multicenter Retrospective Cohort Study in Japan. Lung Cancer, 8, 191-206.

https://doi.org/10.2147/LCTT.S140491 